\title{
Sensory Gating for the Initiation of the Swing Phase in Different Directions of Human Infant Stepping
}

\author{
Marco Y. C. Pang ${ }^{1}$ and Jaynie F. Yang ${ }^{1,2}$ \\ ${ }^{1}$ Centre for Neuroscience and 2 Department of Physical Therapy, University of Alberta, Edmonton, Alberta, Canada \\ T6G 2G4
}

Humans can make smooth, continuous transitions in walking direction from forward to backward. Thus, the processing of sensory input must allow a similar continuum of possibilities. Hip extension and reduced load are two important conditions that control the transition from the stance to swing phase during forward stepping in human infants. The purpose of this study was to determine whether the same factors also regulate the initiation of the swing phase in other directions of stepping. Thirty-seven infants between the ages of 5 and 13 months were studied during supported forward and sideways stepping on a treadmill. Disturbances were elicited by placing a piece of cardboard under the foot and pulling the cardboard in different directions. In this way, the leg was displaced in a particular direction and simultaneously unloaded. We observed whether the swing phase was immediately initiated after the application of disturbances in various directions. Electromyography, vertical ground reaction forces, and hip motion in frontal and sagittal planes were recorded. The results showed that the most potent sensory input to initiate the swing phase depends on the direction of stepping. Although low load was always necessary to initiate swing for all directions of walking, the preferred hip position was always one directly opposite the direction of walking. The results indicated the presence of selective gating of sensory input from the legs as a function of the direction of stepping.

Key words: human; infants; locomotion; proprioceptive input; sensorimotor control; sensory gating
Pattern-generating networks are tremendously flexible, capable of producing different motor behaviors (for review, see Marder and Calabrese, 1996; Marder, 2000). A variety of neuromodulators and input from peripheral and supraspinal sources can modify the neural network to produce different motor patterns (Grillner, 1981; Marder, 1988, 1991; Dickinson, 1989; Getting, 1989; Harris-Warrick, 1991). The same concept has been proposed for the control of locomotion (Grillner, 1981; Clarac, 1984; Pearson, 1993). Direct evidence from the lamprey supports the idea that the same central pattern generator can produce different directions of swimming (Matsushima and Grillner, 1992). Indirect evidence from intact cats suggest that different forms of walking (walk, trot, gallop, upslope, downslope, forward, and backward) might be produced by slight alterations of the same pattern generator (Miller et al., 1975a,b; English, 1979; Buford and Smith, 1990; Buford et al., 1990, 1993; Perell et al., 1993; Carlson-Kuhta et al., 1998). Studies in human infants (Lamb and Yang, 2000) and adults (Thorstensson, 1986; Winter et al., 1989; Earhart et al., 2001) also provided indirect evidence that the same neural circuitry controls different directions of walking.

Sensory input during rhythmic movements are important for controlling phase transitions, such as the transition from stance to swing in walking (for review, see Rossignol, 1996). In different

Received Jan. 22, 2002; revised March 28, 2002; accepted April 15, 2002.

This work was supported by a grant from the Canadian Institutes of Health Research and the Canadian Neurotrauma Research Program to J.F.Y. M.Y.C.P. was supported by a scholarship from the Alberta Heritage Foundation for Medical Research. We thank C. Wolstenholme, F. Lim, and A. t'Hart for technical assistance. We thank Drs. J. Duysens and A. Prochazka for helpful comments on previous versions of this manuscript.

Correspondence should be addressed to Jaynie F. Yang, 2-50 Corbett Hall, University of Alberta, Edmonton, Alberta, Canada T6G 2G4. E-mail: jaynie.yang@ualberta.ca.

Copyright (C) 2002 Society for Neuroscience $0270-6474 / 02 / 225734-07 \$ 15.00 / 0$ directions of walking, however, these sensory signals can be very different at the same phase in the movement. For example, in forward walking, hip extension and reduced load are important sensory signals that promote the initiation of the swing phase (Grillner and Rossignol, 1978; Duysens and Pearson, 1980; Whelan et al., 1995; Hiebert et al., 1996; Whelan and Pearson, 1997; Pang and Yang, 2000, 2001). In backward walking, however, swing phase is initiated when the hip is flexed. How does the pattern generator regulate the stance to swing transition in different directions of walking? In both human infants (Lamb and Yang, 2000) and adults (Stein et al., 1986), there is a continuum of walking directions from forward to backward walking. Smooth transitions between different directions of walking are easily performed (Lamb and Yang, 2000). Hence, the processing of sensory inputs must allow for the infinite number of walking directions and the possibility for smooth transitions between them.

In this study, we focus on how different limb orientations that stretch hip muscles and reduce load affect the initiation of the swing phase in forward and sideways stepping in human infants. We use young infants, because they are much less likely to intervene volitionally with the stepping movements compared with adults. Indeed, their stepping is most likely controlled by circuitry in the spinal cord and brainstem (Forssberg, 1985). Our data show that the direction of the limb motion-orientation that most powerfully promotes the stance to swing transition changes with the direction of walking (i.e., hip extension for forward walking, hip adduction in the leading limb, and hip abduction in the trailing limb for sideways walking). We propose a conceptual model for selective gating of sensory input as a function of walking direction. 


\section{MATERIALS AND METHODS}

Subjects. The infants in this study were recruited through the Public Health Division, Capital Health Authority (Edmonton, Alberta). All of the infants were born at term. Ethical approval was obtained through the Health Research Ethics Board, University of Alberta and the Capital Health Authority (Edmonton, Alberta). Thirty-seven infants aged from 5 to 13 months (mean, 8.4 months) were studied. None of the infants could walk independently. The infant's stepping ability was discussed with a parent. Only those infants who showed 10 consecutive steps at a time, as reported by a parent, were brought in for the experiment. The parent was instructed to practice stepping with the infants for 1-2 min daily for $\sim 1-2$ weeks before the experiment. Previous work has shown practice improves our chances of obtaining good stepping (Yang et al., 1998). Informed and written consent was obtained from a parent before the infant participated in the study. The experiments were conducted in accordance with the Declaration of Helsinki for experiments on human subjects.

Recording procedures. Kendall SOFT-E pediatric $(\mathrm{Ag} / \mathrm{AgCl})$ electrodes were applied over tibialis anterior and gastrocnemius-soleus muscles on each leg after the skin was cleaned with alcohol swabs. A twin-axis electrogoniometer (Penny \& Giles Computer Products, Biometrics, Blackwood Gwent, UK) was placed over the hip joint of the right leg (for 22 infants), the left leg (for 2 infants), or both legs (for 13 infants) to measure hip motion in the sagittal plane (flexion-extension) and the frontal plane (abduction-adduction). The leg recorded from was dependent on the focus of the particular experiment. For example, some experiments focused on comparing the response of the right limb during forward and sideways walking. Others focused on comparing the response of the right and left limb during sideways walking (for details, see Hip disturbances). This was important because infants have a limited tolerance for walking, and only select protocols can be effectively studied in each experiment.

The goniometer was placed so that one arm was aligned with the midaxillary line of the trunk, and the other was along the longitudinal axis of the femur. A video camera (PV-950; Panasonic, Secaucus, NJ) was used to record the left view (for forward walking) or front view (for sideways walking) of the infant. The video record was used qualitatively for identifying good walking sequences.

A Gaitway treadmill system (Kistler Instrument, Amherst, NY) was used for all experiments. Two force plates located beneath the treadmill belt, one in front of the other, were used to measure vertical ground reaction forces during walking. The infant was held under the arms by one of the researchers, with one hand on each side of the infant's upper trunk, allowing the infant to support its own weight as much as possible. The infant was placed on the treadmill at the junction of the two force plates to allow for accurate measurement of load on each leg during stepping. The speed of the treadmill belt was adjusted to obtain optimal stepping in different directions. If possible, several trials of forward and sideways stepping were conducted for each infant. Each trial was typically 1-3 min in length. The whole experimental session took $\sim 1 \mathrm{hr}$. At the end of the session, the weight of the infant was obtained by sitting the infant on the front force plate. Electromyography (EMG), force plate, and electrogoniometer signals were amplified and recorded on VHS tape with a pulse code modulation encoder (A. R. Vetter, Redersburg, PA). All walking trials were videotaped. The video and analog signals were synchronized by a digital counter at a rate of $1 \mathrm{~Hz}$.

Hip disturbances. All of the disturbances were applied manually by a different researcher than the one supporting the infant. The disturbances were intended to stretch a group of hip muscles while simultaneously unloading the leg. A stiff cardboard was placed on the treadmill belt during the swing phase of the limb to be disturbed. After the foot made contact with the cardboard, the leg was dragged in different directions by pulling the cardboard. This allowed us to slide the foot in a direction different from the movement of the treadmill belt. The sliding motion of the cardboard was done as quickly as possible in each direction. The infants were always distracted with toys and games throughout the walking trials. Most infants did not seem to notice the application of the disturbances.

In forward stepping, all perturbations were applied to the right leg. Normally, the hip is extended at swing phase initiation in forward walking. We wanted to test whether stretching the hip in the opposite direction (flexion) or a direction orthogonal to the direction of progression (abduction or adduction) would promote or hamper the initiation of the swing phase. Therefore, the right hip was perturbed in four different directions in forward stepping: flexion, extension, abduction, and adduction. In each case, the limb was unloaded by the disturbance.

In sideways stepping, the right leg was always the leading leg in these experiments. The hip of the leading leg is normally adducted at the time swing phase is initiated. We were interested in determining whether hip movement in the opposite direction (abduction) would be less effective in initiating the swing phase. In addition, we wanted to determine whether hip extension, the powerful trigger to initiate swing phase in forward stepping, was also effective in sideways stepping. Therefore, three different directions of disturbances were applied to the leading limb: extension, abduction, and adduction. Again, the limb was unloaded by the disturbance.

In contrast to the leading limb, the trailing (left) limb normally initiates its swing phase when the hip is abducted during sideways walking. Because hip movements of the leading and trailing limbs are opposite, abduction and adduction disturbances were also applied to the trailing limb to determine whether the leading and trailing limb reacted differently to the two directions of disturbances.

The disturbances described so far involved stretching a group of hip muscles together with unloading the stance leg. We also wanted to study the reactions of the leg when the load was high and the hip angle was in a neutral position. Therefore, during both forward and sideways walking, another type of disturbance was used, in which the right hip was kept in a neutral position by holding the cardboard stationary once the limb reached the midstance phase (called mid-disturbances). In other words, we prevented the normal hip extension (forward walking) or hip adduction (sideways walking, leading limb) from occurring. Again, we observed whether the swing phase was initiated after the application of these disturbances.

Data analysis. The data were analyzed off-line. The EMG data were high-pass filtered at $10 \mathrm{~Hz}$, full-wave rectified, and low-pass filtered at 30 $\mathrm{Hz}$. The force plate and the electrogoniometer signals were also low-pass filtered at $30 \mathrm{~Hz}$. All of the signals were then analog-to-digitally converted at $250 \mathrm{~Hz}$ (Axoscope 7; Axon Instruments, Foster City, CA).

The video data were reviewed to identify good sequences of walking and successful disturbances (for definition, see below). The corresponding analog data were then identified. The stance and swing phase durations were estimated by the time of right foot contact and toe off, respectively, using the force plate signals in conjunction with the video image. All of the undisturbed steps were selected and averaged using a customized software program.

The EMG, force plate, and goniometer signals from undisturbed steps were aligned at the time of foot-ground contact. Average profiles were produced for a cycle of stepping. The mean force value over the averaged step cycle gave us an estimate of the amount of weight the infant was bearing during stepping.

The time at which swing phase was initiated was determined by the reversal of the hip goniometer signal from extension to flexion for both directions of stepping. This is reasonable, because, when swing phase was initiated in sideways stepping, the reversal of hip motion from adduction to abduction (leading limb) and from abduction to adduction (trailing limb) corresponded well to the reversal from extension to flexion. The corresponding load at the time swing phase was initiated was estimated by the force plate signal.

For all types of disturbances (except mid-disturbances), the beginning of the disturbance was indicated by a sudden change in the goniometer signal in the intended direction. The end of the disturbance was defined as the time when the goniometer signal reached a peak in the intended direction. The angular velocity of hip movement during the perturbation and the duration of the disturbance could thus be computed. The disturbance was considered successful if (1) it was preceded and followed by a complete step, (2) the hip angle between the beginning and the end of the disturbance had a difference of $>10^{\circ}$, and (3) the angular speed of the hip motion was higher than $30^{\circ} / \mathrm{sec}$ in the intended direction.

For each type of disturbance, the disturbed leg would react by (1) initiating the swing phase or (2) continuing its stance phase and not initiating the swing phase until much later. The latency for the initiation of the swing phase was defined as the time period from the beginning of the disturbance to the beginning of the hip flexion movement associated with the swing phase. Because the duration of the majority of disturbances ranged from 200 to $500 \mathrm{msec}$, the swing phase was considered to be successfully initiated if it occurred within $700 \mathrm{msec}$ after the onset of the perturbation. The percentage of trials in which the swing phase was successfully initiated was determined for each type of disturbance. This value was then compared across different groups of disturbances. 

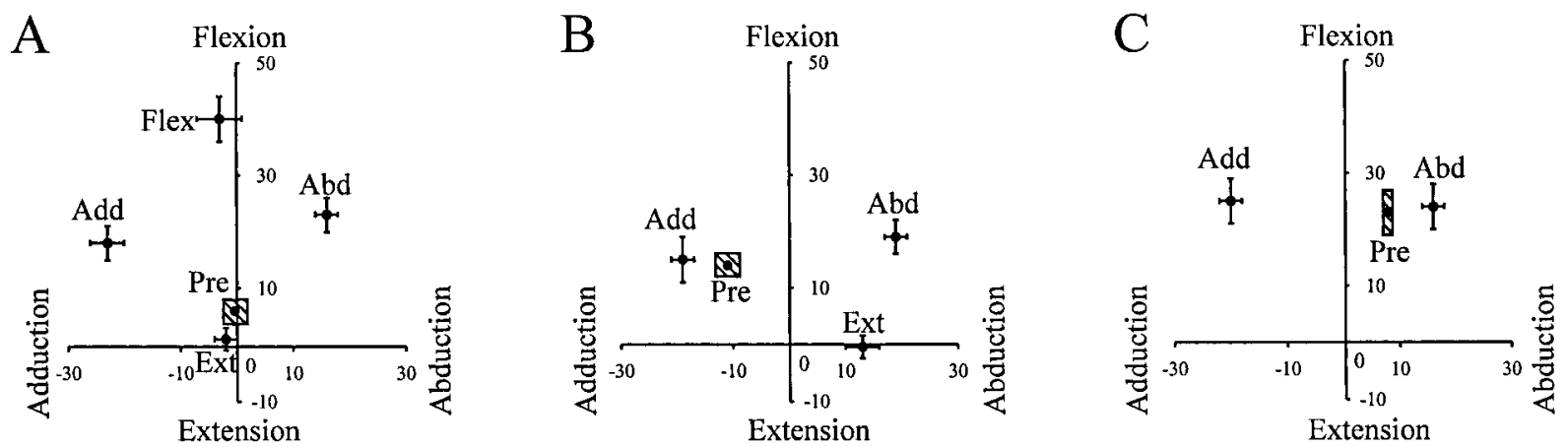

Figure 1. Pooled data: hip angle at the end of disturbances. The final hip angle achieved at the end of disturbances is shown for forward walking $(A)$, the leading limb in sideways walking $(B)$, and the trailing limb in sideways walking $(C)$. The horizontal axis represents the hip abduction-adduction angle, and the vertical axis represents hip flexion-extension angle. The label beside each data point represents the direction of the disturbance: Flex, flexion; Ext, extension; Abd, abduction; Add, adduction. The error bar represents one SE. The shaded box labeled Pre represents one SE for the normal steps preceding the disturbance. The data show that we were successful in altering the hip angle in the intended directions.

Statistical analysis. For each type of disturbance, paired $t$ tests were used to compare the hip angle and load at swing initiation for the predisturbed step and those at the end of the disturbance. This was done to make sure that the disturbances applied were effective in changing the hip angle in the intended direction and reducing the load (or increasing the load for mid-disturbances). For comparison of data (i.e., hip angle, load, and hip angular velocity of disturbances) between more than two types of disturbances in a given direction of walking or in a given limb in sideways walking, one-way ANOVAs were used. Bonferroni's $t$ tests were conducted to compare the data post hoc. To compare the data between two types of disturbances, independent sample $t$ tests were used. All statistical tests were conducted with mean values from each subject (i.e., averaged across all successful trials). In addition, to compare the proportion of successful trials in eliciting swing phase among different categories of disturbances, $\chi^{2}$ test of association was used. A probability level of 0.05 for type I error was set for all statistical tests. For post hoc $t$ tests, the probability level was adjusted depending on the number of comparisons so as to reduce the probability of making a type I error (Glass and Hopkins, 1996).

\section{RESULTS}

Of the 37 subjects, 22 subjects participated in both directions of walking. Seven and eight subjects participated only in forward or sideways walking, respectively. For all trials, the speed of the treadmill ranged from 0.23 to $0.27 \mathrm{msec}^{-1}$. In forward walking, the amount of weight borne by the infants during stepping ranged from 28 to $57 \%$ of their own body weight (BW) (mean, $48 \% \mathrm{BW}$ ). In sideways walking, the amount of weight borne by the infants ranged from 16 to $53 \% \mathrm{BW}$ (mean, $31 \% \mathrm{BW}$ ). For those subjects who participated in both directions of walking, the amount of weight borne during forward walking was significantly greater than that during sideways walking.

Statistical analysis revealed that all types of disturbances were effective in causing a significant deviation of the hip angle in the intended direction when compared with the predisturbed steps (Fig. 1). Moreover, the deviation in the hip angle for each type of disturbance was significantly different from each other in the intended direction. For example, in forward walking, the hip angle was significantly more flexed after flexion disturbances than that after the other three types of disturbances (Fig. 1A). Note that infants adopt a more flexed posture than adults, and the hip rarely extends past the neutral position. In sideways walking, disturbances to the leading and trailing limbs are shown in Figure $1, B$ and $C$, respectively. Disturbances in the extension direction included some abduction, because it was important to elicit the extension before the leading limb met the trailing limb at midline.

After the application of different disturbances, there were two

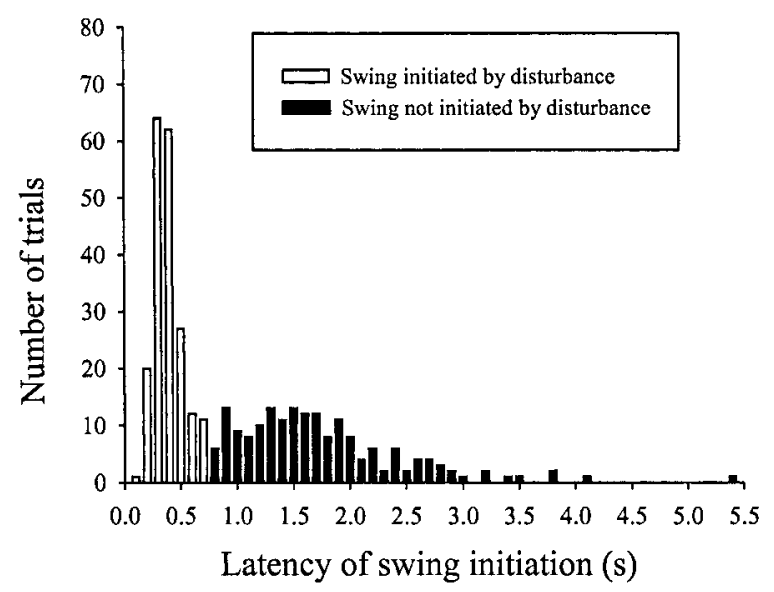

Figure 2. Latencies for the initiation of the swing phase. The open bars represent those trials in which the swing phase was successfully initiated by the disturbance (within $700 \mathrm{msec}$ after onset of the perturbation). The black bars represent those trials in which the disturbed leg continued with its stance phase after the disturbance and did not initiate its swing phase until much later.

types of responses: (1) the limb initiated the swing phase immediately, or (2) the limb continued its stance phase and did not initiate the swing phase until much later. The distribution of latencies for the initiation of the swing phase is shown in Figure 2. The swing phase was successfully initiated in 197 of 373 trials. For each direction of disturbance, the characteristics of the disturbances (i.e., hip angle, load, and speed) were compared between the two types of response. No significant difference was found. Therefore, the data for each type of disturbance were pooled regardless of the response and then analyzed.

\section{The most powerful input to initiate swing phase changes with direction of walking}

The most potent sensory input to promote swing phase initiation was a function of the direction of walking (Fig. 3). In forward walking, the most powerful sensory input to trigger the onset of the swing phase was hip extension. Stretching the hip in the opposite direction (i.e., flexion) or in a direction orthogonal to that of progression (i.e., abduction and adduction) resulted in a significantly lower success rate of initiating the swing phase when compared with hip extension (Fig. 3, open bars). The results thus indicated that, as long as the hip was kept from reaching an 


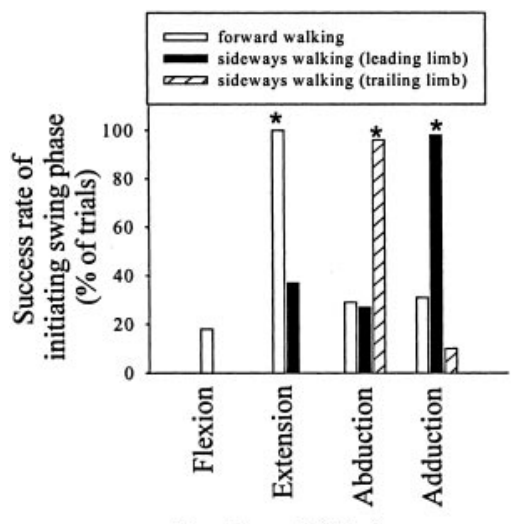

Direction of disturbances

Figure 3. Success rate of initiating the swing phase. In forward walking, hip extension was the most powerful sensory input to initiate the swing phase. In sideways walking, hip adduction and hip abduction became the most potent input to induce the onset of the swing phase in the leading limb and trailing limb, respectively. Asterisks represent a statistically significant difference from the other types of disturbances in the same direction of walking or in the same limb (for sideways walking).

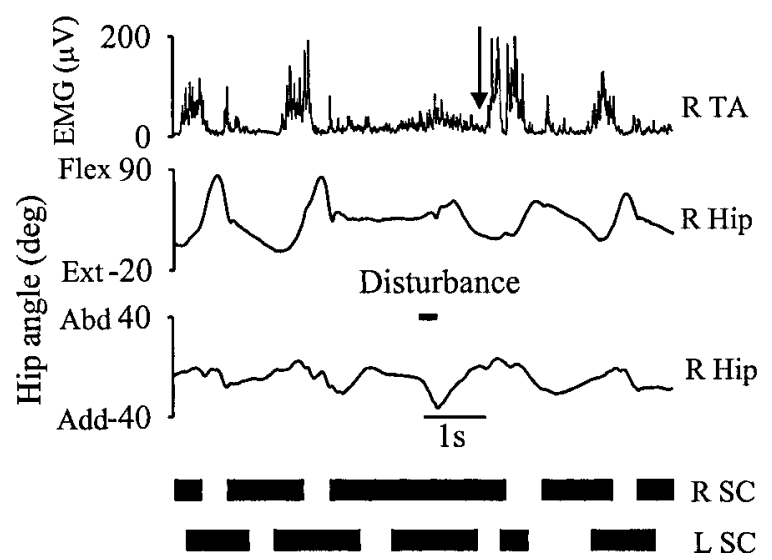

Figure 4. Example of an adduction disturbance in forward walking. Surface electromyography from the right tibialis anterior, goniometer measures of the right hip, and step cycles of both legs are shown for a single subject (LG). The thick line between the second and the third traces represents the duration of the disturbance. The black bars at the bottom of the graph represent stance phase, whereas the space between the bars represents swing phase. In this particular example, the right hip was adducted to $-33^{\circ}$ by the disturbance. Swing phase was not initiated until much later, when the hip reached an extended position (see arrow in $R T A$ signal and the corresponding hip flexion seen in the goniometer signal). The right stance phase was prolonged as a result. $T A$, Tibialis anterior; $S C$, step cycle; $R$, right; $L$, left; Flex, flexion; $E x t$, extension; $A b d$, abduction; $A d d$, adduction.

extended position during forward stepping, adding an abductionadduction component did not significantly increase the probability of initiating the swing phase.

An example of an adduction disturbance in forward walking is shown in Figure 4. In all figures, positive numbers in the joint angles represent flexion and abduction. The right hip was adducted to $-33^{\circ}$. The limb did not initiate the swing phase until much later when it reached an extended position [see arrow in right tibialis anterior $(R T A)$ EMG signal and the corresponding goniometer signal].

Interestingly, hip extension, which was the most powerful sensory input to initiate the swing phase in forward walking, became relatively ineffective in inducing swing phase in sideways walking.
Hip adduction became the most effective input to promote swing phase initiation in the leading limb (Fig. 3, solid bars). Similar to the situation in forward walking, adding an orthogonal component (extension) to the leading limb did not increase the likelihood of initiating the swing phase, provided that the hip was not able to attain adduction. An example of an adduction disturbance to the leading limb in sideways walking is shown in Figure $5 A$. The hip was adducted to $-13^{\circ}$. The leg reacted to the disturbance by initiating the swing phase [see early onset of EMG burst in the right tibialis anterior muscle after the disturbance (Fig. $5 A$, arrow in top trace)].

Sideways walking is different from forward walking in that the hip motion of the two legs are opposite in the same phase of the walking cycle. For example, during stance phase, the hip adducts in the leading limb, whereas the hip abducts in the trailing limb. Interestingly, the leading and the trailing leg also showed opposite responses to the different directions of disturbances. Adduction disturbances in the trailing limb typically did not lead to the immediate initiation of swing phase. Figure $5 B$ shows an example of such a disturbance in the same infant as that shown in Figure $5 A$. The hip is adducted more $\left(-32^{\circ}\right)$ in the trial shown in Figure $5 B$ compared with Figure $5 A$. Despite that, initiation of the swing phase was delayed. Group data shown in Figure 3 (cross-hatched bars) indicates that abduction disturbances were far more likely to initiate swing phase than adduction disturbances in the trailing limb.

Could the effects described above be explained by the difference in the amount of unloading and speed of disturbance among different types of disturbances? Our results showed that this possibility was very unlikely. Pooled data for the load at the end of disturbances and the angular speed of hip motion during the disturbances are shown in Figure 6. There was no significant difference in load and speed among the different types of disturbances in a given direction of walking or in a given limb (for sideways walking). Therefore, the results could not be explained by systematic differences in the amount of unloading or the speed of disturbances.

\section{Responses to mid-disturbances}

All of the results reported so far concerned different directions of hip motion coupled with unloading. We reported previously that, during forward walking, if the limb was kept in a midstance position, with a neutral hip angle and high load on the limb, swing phase was held off indefinitely (Pang and Yang, 2000). The same was found in sideways walking (Fig. 7). During the course of the disturbance, the leading limb was maintained in a slightly abducted position. The disturbed limb remained in the stance phase while the contralateral leg continued to step (reflected in the ongoing rhythmic activity in left tibialis anterior signal).

\section{DISCUSSION}

The response to various directions of disturbances was highly dependent on the direction of walking. The most effective sensory input to promote swing phase initiation for a given direction of walking was leg motion in a direction opposite to that of progression. Interestingly, this meant that, in sideways walking, the reactions in the leading limb were opposite to the trailing limb. Although the powerful effects of hip extension to initiate the swing phase in forward walking are well established in both animal (Grillner and Rossignol, 1978; Hiebert et al.,1996) and human infant (Pang and Yang, 2000, 2001) studies, the different 
Figure 5. Example of abduction and adduction disturbance to the leading and trailing limbs in sideways walking. Data in both parts of the figure are from the same infant (subject EO). The convention of this figure is the same as Figure 4. $A$, Response of the leading leg to a disturbance that adducted the hip to $-13^{\circ}$. The swing phase was initiated early (see arrow in $R T A$ signal). $B$, Response of the trailing leg to a disturbance that adducted the hip to $-32^{\circ}$. In this case, swing phase was delayed. $T A$, Tibialis anterior; $S C$, step cycle; $R$, right; $L$, left; Flex, flexion; Ext, extension; $A b d$, abduction; $A d d$, adduction.
A
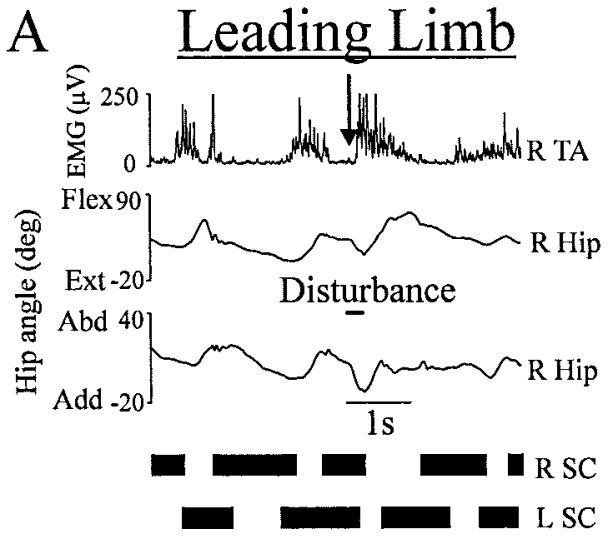
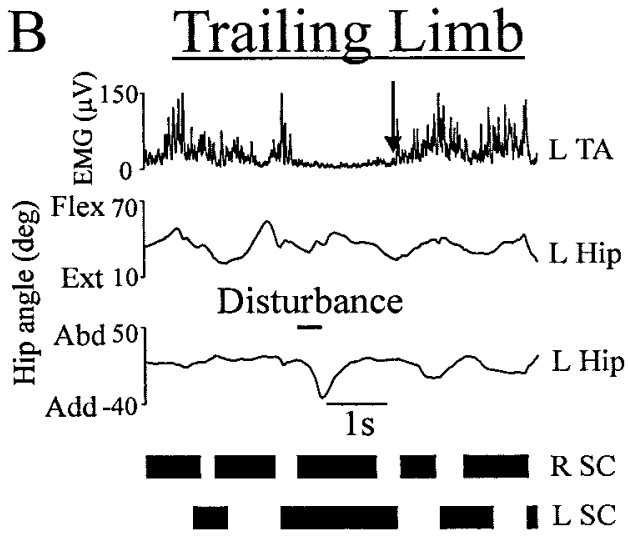

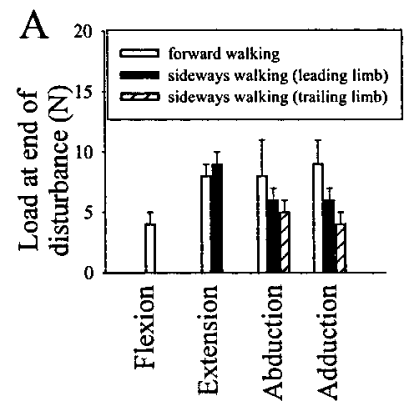

Direction of disturbances

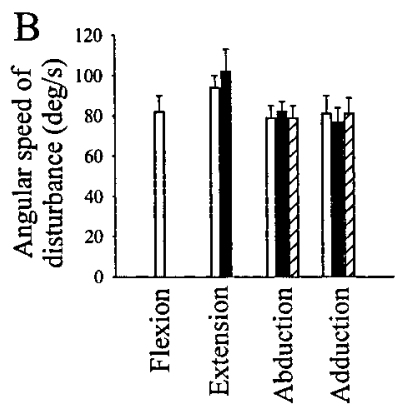

Direction of disturbances

Figure 6. Pooled data: load at the end of disturbances and speed of disturbances. $A$, Load at the end of disturbances. $B$, The angular speed of the hip motion during the disturbances. There was no statistically significant difference in load and speed among the different types of disturbances in a given direction of walking or in a given limb (for sideways walking).

reactions of the leading and the trailing legs during sideways walking are now shown for the first time.

\section{Methodological considerations}

All of the disturbances in this study were applied manually. Variability between disturbances was thus unavoidable. The data showed, however, that we were successful in inducing hip angle changes in the intended direction in all cases, with limited variation (Fig. 1). Moreover, other variables that might have affected the success rate for swing phase initiation, such as load on the limb and the angular speed of hip movement, were not significantly different for the different directions of disturbances (Fig. 6).

The disturbances displaced the whole lower limb in a particular direction. The induced movements were not confined to the hip, and many types of sensory afferents could have been activated. The limb position at the end of the disturbances, however, were generally very similar for all directions of disturbances (knee extended and ankle plantarflexed), except for the position of the hip. Thus, we feel it is likely that afferents signaling hip position would reflect the different directions of disturbances most. For simplicity in the discussion, we will refer to hip motion when discussing different directions of disturbances. In reality, the results could be explained equally well by a convergence of signals that reflect the limb orientation (Bosco and Poppele, 2001).

The amount of weight borne by the infants during sideways walking was $36 \%$ less than that during forward walking. These differences in weight bearing do not interfere with our interpretation, because the comparisons we made were confined to the
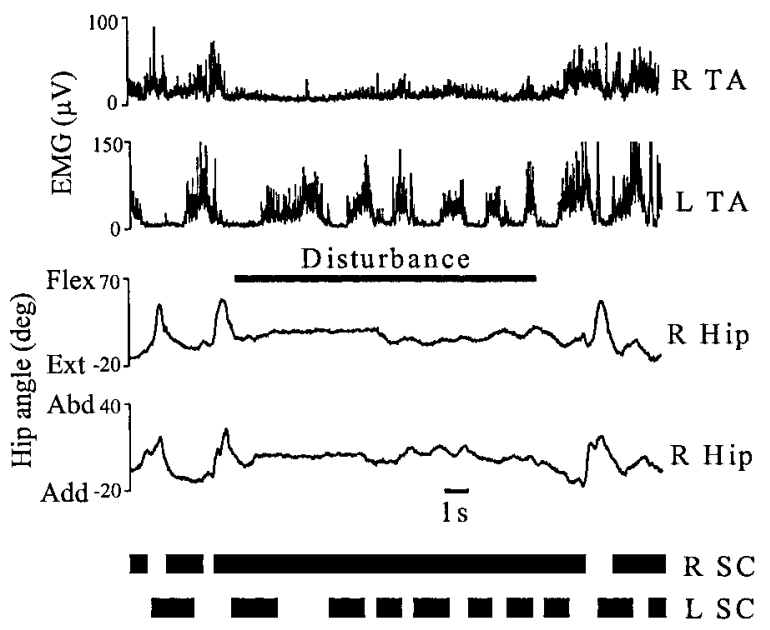

Figure 7. An example of a mid-disturbance in sideways walking. Responses from a single subject (ML). Convention of the figure is the same as Figure 4 . The hip was kept in a slightly abducted position (mean, $4^{\circ}$ ), and the load was high (mean, $28 \mathrm{~N}$; data not shown). As a result, the stance phase was prolonged. The normal alternating activity of the right tibialis anterior was terminated. In contrast, the left leg continued to step (see ongoing alternating activity in the left tibialis anterior). TA, Tibialis anterior; $S C$, step cycle; $R$, right; $L$, left; Flex, flexion; Ext, extension; $A b d$, abduction; $A d d$, adduction.

relative ease with which swing phase was initiated in each direction of walking. We do not compare the unloading necessary for swing initiation in different directions of walking.

\section{Central pattern generators for different directions of walking}

Before discussing the nature of sensory gating during different directions of walking, we must first consider whether the same pattern generator controls the different directions of walking. This question has been addressed indirectly in the human adult. Based on comparisons of the muscle activation patterns and the movement patterns, some have suggested that there is sufficient similarity in the patterns to suggest that the same pattern generator is involved (Thorstensson, 1986; Winter et al., 1989; Grasso et al., 1998). More recently, Earhart et al. (2001) showed that, after walking on the perimeter of a rotating disk, the curved locomotor trajectories of forward and backward walking over stationary ground were very similar. This transfer of learning to both directions of walking supports the idea that forward and backward walking are controlled by similar neural circuitry.

Adult humans, however, can volitionally intervene with the 
stepping movements, making it difficult to determine the contributions of the lower brain centers to the control of different directions of walking. Because young infant have far less descending control (Forssberg, 1985; Yang et al., 1998), their stepping provides more direct information about the pattern generator. Recent results from our laboratory support the idea that the same pattern generator controls different directions of stepping in young infants (Lamb and Yang, 2000). We showed that the majority of infants can step in all directions from the time forward stepping is expressed. Moreover, changes to the stance and swing phase durations vary in the same way for all directions of walking, and infants can change their stepping in a continuous way from forward to backward.

\section{Selective gating of sensory input}

Our current results show that the same sensory input produces a very different response that is a function of the walking direction. Thus, there must be gating of sensory input that is a function of the walking direction. Based on the assumption that the same locomotor pattern generator controls all directions of walking, the experimental evidence presented in this paper predicts that there is a large convergence of sensory input to the pattern generator. Afferents that signal various hip positions (or limb orientations), for example, should all have access to the pattern generator. Depending on the direction of walking, the gains in these pathways are selectively altered.

Using the half-center model for locomotion (Brown, 1911, 1914; Lundberg, 1980), we propose a conceptual model in which there is convergence of sensory input from the legs to the flexor and extensor half-center. Our data suggest that load always influences the decision to initiate swing phase, regardless of the direction of walking. Afferents signaling hip position (or limb orientation) are selectively gated as a function of the walking direction. For example, in forward walking, gains in reflex pathways from stretch-sensitive afferents in hip flexor muscles are higher than those from other hip muscles. During sideways walking, the reflex gains from stretch-sensitive afferents in hip abductors to the pattern generator are higher in the leading limb, whereas those from hip adductors are higher in the trailing limb. We further predict that, with walking directions intermediate between forward and sideways, the reflex gains from stretchsensitive afferents in hip flexors and abductors of the leading limb would be higher than those from other hip muscles. Thus, the most effective stretch to initiate the swing phase in this case would be a combined hip extension and adduction (i.e., directly opposite to the direction of walking).

Previous studies have shown that transmission in reflex pathways is highly dependent on the form of the task (for review, see Rossignol, 1996). For example, the phase-dependent responses to cutaneous stimulation of the leg in forward walking are different from those in backward walking (Buford and Smith, 1993; Duysens et al., 1996). Based on the pattern of reflex modulation, Duysens et al. (1996) suggested that their results from backward walking could be explained by a reversal of the motor program that produces forward walking. No details were given regarding how this might be achieved. Our current results suggest that we must consider the changes in reflex gain from forward to backward walking as a continuum, which must account for all directions of walking in-between. With this in mind, the models proposed must also reflect the continuum of walking directions.

Together, these results lend support to the idea that the human nervous system uses sensory input in a probabilistic way to make motor decisions. The relative weighting of many different sensory inputs are used in the final decision (Bassler, 1993; Prochazka, 1996a,b; Prochazka and Yakovenko, 2001). In this way, sensory input related to different directions of hip motion all contribute to the motor decision of whether or not to initiate the swing phase. Indeed, swing phase can still be initiated when the hip position was not optimal (Fig. 3) but at a reduced probability.

To our knowledge, this is the first report to show that different sensory signals control the stance to swing transition for different directions of walking in humans. The results indicate the presence of selective gating of sensory input as the direction of walking changes. We further predict that the mechanism for selecting the sensory signals must form a continuum to account for the continuum of walking directions possible in humans.

\section{REFERENCES}

Bassler U (1993) The femur-tibia control system of stick insects-a model system for the study of the neural basis of joint control. Brain Res Rev 18:207-226.

Bosco G, Poppele RE (2001) Proprioception from a spinocerebellar perspective. Physiol Rev 81:539-568.

Brown TG (1911) The intrinsic factors in the cat of progression in the mammal. Proc R Soc Lond B Biol Sci 84:308-319.

Brown TG (1914) On the nature of the fundamental activity of the nervous centres; together with an analysis of the conditioning of rhythmic activity in progression, and a theory of the evolution of function in the nervous system. J Physiol (Lond) 48:18-46.

Buford JA, Smith JL (1990) Adaptive control of backward quadrupedal walking. II. Hindlimb muscle synergies. J Neurophysiol 64:756-766.

Buford JA, Smith JL (1993) Adaptive control for backward quadrupedal walking. III. Stumbling corrective reactions and cutaneous reflex sensitivity. J Neurophysiol 70:1102-1114.

Buford JA, Zernicke RF, Smith JL (1990) Adaptive control for backward quadrupedal walking. I. Posture and hindlimb kinematics. J Neurophysiol 64:745-755.

Carlson-Kuhta P, Trank TV, Smith JL (1998) Forms of forward quadrupedal locomotion. II. A comparison of posture, hindlimb kinematics, and motor patterns for upslope and level walking. J Neurophysiol 79:1687-1701.

Clarac F (1984) Spatial and temporal co-ordination during walking in Crustacea. Trends Neurosci 7:3293-3298.

Dickinson P (1989) Modulation of simple motor patterns. Semin Neurosci $1: 15-24$.

Duysens J, Pearson KG (1980) Inhibition of flexor burst generation by loading ankle extensor muscles in walking cats. Brain Res 187:321-332.

Duysens J, Tax AAM, Murrer L, Dietz V (1996) Backward and forward walking use different patterns of phase-dependent modulation of cutaneous reflexes in humans. J Neurophysiol 76:301-310.

Earhart GM, Melvill Jones G, Horak FB, Block EW, Weber KD, Fletcher WA (2001) Forward versus backward walking: transfer of podokinetic adaptation. J Neurophysiol 86:1666-1670.

English AW (1979) Interlimb coordination during stepping in the cat: an electromyographic analysis. J Neurophysiol 42:229-243.

Forssberg H (1985) Ontogeny of human locomotor control. I. Infant stepping, supported locomotion and transition to independent locomotion. Exp Brain Res 57:480-493.

Getting PA (1989) Emerging principles governing the operation of neural networks. Annu Rev Neurosci 12:185-204.

Glass GV, Hopkins KD (1996) Statistical methods in education and psychology, Ed 3. Needham Heights, MA: Allyn \& Bacon.

Grasso R, Bianchi L, Lacquaniti F (1998) Motor patterns for human gait: backward versus forward locomotion. J Neurophysiol 80:1868-1885.

Grillner S (1981) Control of locomotion in bipeds, tetrapods, and fish. In: Handbook of physiology, Sec 1, The nervous system, Vol II (Brookhart JM, Mountcastle VB, eds), pp 1179-1236. Bethesda, MD: American Physiological Society.

Grillner S, Rossignol S (1978) On the initiation of the swing phase of locomotion in chronic spinal cats. Brain Res 146:269-277.

Harris-Warrick RM (1991) Modulation of neural networks for behavior. Annu Rev Neurosci 14:39-57.

Hiebert GW, Whelan PJ, Prochazka A, Pearson KG (1996) Contribution of hindlimb flexor muscle afferents to the timing of phase transitions in the cat step cycle. J Neurophysiol 81:758-770.

Lamb T, Yang JF (2000) Could different directions of infant stepping be controlled by the same locomotor central pattern generator? J Neurophysiol 83:2814-2824.

Lundberg A (1980) Half-centres revisited. In: Advanced physiological science, Vol 1, Regulatory functions of the CNS. Motion and organi- 
zation principles (Szentagothai J, Palkovitis M, Hamori J, eds), pp 155-167. New York: Pergamon.

Marder E (1988) Modulating a neural network. Nature 335:296-297.

Marder E (1991) Modifiability of pattern generation. Curr Opin Neurobiol 1:571-576.

Marder E (2000) Motor pattern generation. Curr Opin Neurobiol 10:691-698.

Marder E, Calabrese RL (1996) Principles of rhythmic motor pattern generation. Physiol Rev 76:687-717.

Matsushima T, Grillner S (1992) Neural mechanisms of intersegmental coordination in lamprey: local excitability changes modify the phase coupling along the spinal cord. J Neurophysiol 67:373-388.

Miller S, Van Der Burg J, Van Der Meche FGA (1975a) Coordination of movements of the hindlimbs and forelimbs in different forms of locomotion in normal and decerebrate cats. Brain Res 91:217-237.

Miller S, Van Der Burg J, Van Der Meche FGA (1975b) Locomotion in the cat: basic programmes of movement. Brain Res 91:239-253.

Pang MYC, Yang JF (2000) The initiation of the swing phase in human infant stepping: importance of hip position and leg loading. J Physiol (Lond) 528:389-404.

Pang MYC, Yang JF (2001) Interlimb coordination in human infant stepping. J Physiol (Lond) 533:617-625.

Pearson KG (1993) Common principles of motor control in vertebrates and invertebrates. Annu Rev Neurosci 16:265-297.

Perell KI, Gregor RJ, Buford JA, Smith JL (1993) Adaptive control for backward quadrupedal walking. IV. Hindlimb kinetics during stance and swing. J Neurophysiol 70:2226-2240.

Prochazka A (1996a) Proprioceptive feedback and movement regulation. In: Handbook of physiology, Sec 12, Exercise: regulation and integration of multiple systems (Rowell L, Sheperd JT, eds), pp 89-127. New York: Oxford UP.

Prochazka A (1996b) The fuzzy logic of visuomotor control. Can J Physiol Pharmacol 74:456-462

Prochazka A, Yakovenko S (2001) Locomotor control: from spring-like reactions of muscles to neural prediction. In: The somatosensory system: deciphering the brain's own body image (Nelson R, ed), pp 141-181. Boca Raton, FL: CRC.

Rossignol S (1996) Neural control of stereotypic limb movements. In: Handbook of physiology, Sec 12, Exercise: regulation and integration of multiple systems (Rowell L, Sheperd JT, eds), pp 173-216. New York: Oxford UP.

Stein PSG, Mortin LI, Robertson GA (1986) The forms of a task and their blends. In: Neurobiology of vertebrate locomotion, pp 201-216. London: Macmillan.

Thorstensson A (1986) How is the normal locomotor pattern modified to produce backward walking? Exp Brain Res 61:664-668.

Whelan PJ, Pearson KG (1997) Comparison of the effects of stimulating extensor group I afferents on cycle period during walking in conscious and decerebrate cats. Exp Brain Res 117:444-452.

Whelan PJ, Hiebert GW, Pearson KG (1995) Stimulation of the group I extensor afferents prolongs the stance phase in walking cats. Exp Brain Res 103:20-30.

Winter DA, Pluck N, Yang JF (1989) Backward walking: a simple reversal of forward walking? J Mot Behav 21:291-305.

Yang JF, Stephens MJ, Vishram R (1998) Infant stepping: a method to study the sensory control of human walking. J Physiol (Lond) 507:927937. 